\title{
Management of an Oblique Crown Fracture of an Anterior Tooth with a Missed Coronal Segment: A Case Report
}

\author{
Merin Joseph ${ }^{1}$, Radhakrishnan Nair K², Praveena Geetha ${ }^{3}$, Rakhi P Veedu ${ }^{4}$, Emi George ${ }^{5}$
}

\begin{abstract}
Background: Coronal fractures of the anterior teeth are a common form of dental trauma that mainly affects children and adolescents. One of the options for managing coronal tooth fractures when the tooth fragment is available and there is no or minimal violation of the biological width is the reattachment of the dental fragment. Sometimes the reattachment alone does not give favorable esthetic result if a fragment is missing and composite restoration may be combined with reattachment for restoring the crown morphology.

Case description: This case report describes the management of a complicated oblique crown fracture of a maxillary right central incisor. The mobile fractured segment was removed under local anesthesia (LA) and stored in normal saline. Endodontic treatment was given with a master cone size of 50. Reattachment of the tooth fragment was done using the dual-cure resin cement. Access cavity and missing portion of the tooth were restored with composite resin to regain the tooth morphology.

Conclusion: In this case, oblique crown fracture of a young patient was effectively managed with a single-visit endodontic treatment followed by reattachment of tooth fragment. The missing coronal part was restored with composite which gives a better reinforcement to the fractured segment and the remaining tooth structure.

Clinical significance: Reattachment of the tooth is a viable treatment alternative to crowning in anterior tooth trauma for a young patient. It is a viable technique that restores function and esthetics with a very conservative approach and can be considered as a choice when treating a patient with coronal fractures of the anterior teeth especially in a young patient.

Keywords: Missed coronal segment, Oblique crown fracture, Reattachment.

Conservative Dentistry and Endodontic Journal (2019): 10.5005/jp-journals-10048-0044
\end{abstract}

\section{BACKGROUND}

Traumatic dental injuries are the most common type of injury occurring in an individual which usually occur in young people aged 6-13 years. ${ }^{1}$ Coronal fractures represent a higher proportion of dental trauma in the permanent dentition ranging from 26 to $76 \%$ of dental injury and approximately $16 \%$ of coronal fractures are complicated. In coronal fractures, maxillary central incisors are the most commonly affected teeth followed by maxillary lateral incisors and mandibular incisors. ${ }^{2}$ The main causes of trauma that affect the permanent incisors are falls, collisions, sports, violence, and road traffic accidents. It has a severe impact on the social and psychological wellbeing of the patient.

Among coronal fractures, oblique crown fractures are most difficult to manage due to its involvement into the subgingival area. Several factors influence the management of oblique coronal tooth fractures, including the extent of the fracture, biological width violation, endodontic involvement, alveolar bone fracture, restorability of fractured tooth, presence or absence of fractured tooth fragment, and its condition for use. . $^{3,4}$ Treatment options to restore the fractured crown range from simple composite resin restoration to jacket crown. One of the options for managing coronal tooth fractures is the reattachment of the dental fragment when it is available. ${ }^{5}$

It is a simple procedure which can provide good and longlasting esthetics and restore function. Clinical trials have reported that reattachment using an adhesive luting system achieve reasonable functional and esthetic success. ${ }^{6}$ Sometimes the reattachment alone does not give favorable aesthetic result if a fragment is missing and composite restoration may be combined with reattachment for restoring the crown morphology. This case

\footnotetext{
${ }^{1-5}$ Department of Conservative Dentistry and Endodontics, Azeezia College of Dental Sciences and Research, Kollam, Kerala, India
}

Corresponding Author: Merin Joseph, Department of Conservative Dentistry and Endodontics, Azeezia College of Dental Sciences and Research, Kollam, Kerala, India, Phone: +91 9447258195, e-mail: merinjoseph246@gmail.com

How to cite this article: Joseph M, Nair KR, et al. Management of an Oblique Crown Fracture of an Anterior Tooth with a Missed Coronal Segment: A Case Report. Cons Dent Endod J 2019;4(1):21-24.

Source of support: Nil

Conflict of interest: None

report describes a single-visit management of a complicated oblique crown fracture of a maxillary central incisor.

\section{Case Description}

A 13-year-old male came to the department with a complaint of the fractured upper front tooth with associated pain and difficulty in mouth closure. Dental history revealed that the trauma was a result of a fall on the previous day during sports' activities. He had a swollen upper lip with lacerations. Medical history was noncontributory. Intraoral examination revealed an Ellis class III fracture of 11 with a palatally displaced fractured segment on the distal aspect of the tooth with a missing coronal segment incisally (Fig. 1). The line of fracture was oblique, the segment was mobile, and the tooth was tender on percussion. Radiographic examination showed a fracture line approximating the pulp and periapical area was normal (Fig. 2). The diagnosis was a complicated oblique 


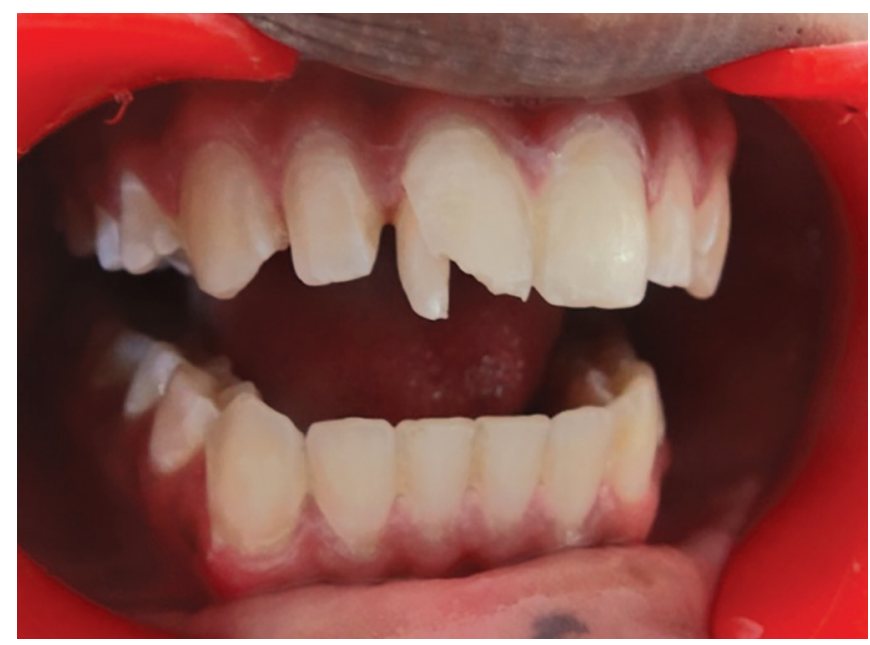

Fig. 1: Preoperative photograph

crown fracture on 11. No mobility of anterior teeth was recorded and surrounding intraoral soft tissues and alveolar bone were normal. Various treatment options were presented to the patient, regarding their advantages, disadvantages, cost, and prognosis. Reattachment option was presented only after confirming that the fragments were in good condition and that they fit reasonably well on the fractured teeth.

The treatment plan was a single-visit endodontic treatment followed by reattachment of the fractured teeth. The mobilefractured segment was removed under LA and stored in normal saline. Irrigation was done with $5 \%$ sodium hypochlorite (Associated Clinical Aids, India) and normal saline (Eurolife Health Care, India) along with cleaning and shaping. Obturation was done with a master cone size of 50 (Fig. 3). After obturation, laser gingivectomy was performed to expose the palatal aspect of the crown margin (Fig. 4). The fragment was then assessed for placement in the original position. The margins of the tooth and fragment were well fitting. Both the fragment and the tooth were etched with a nonrinse conditioner (Parabond, Coltene). A bonding agent (Parabond, Coltene) was applied in 2 coats to both the substrates, gently air thinned, and light cured for 15 seconds. Tooth fragment was attached using a dual-cure resin cement (Paracore, Coltene) with no preparation technique (Figs 5 and 6). Excess resin cement

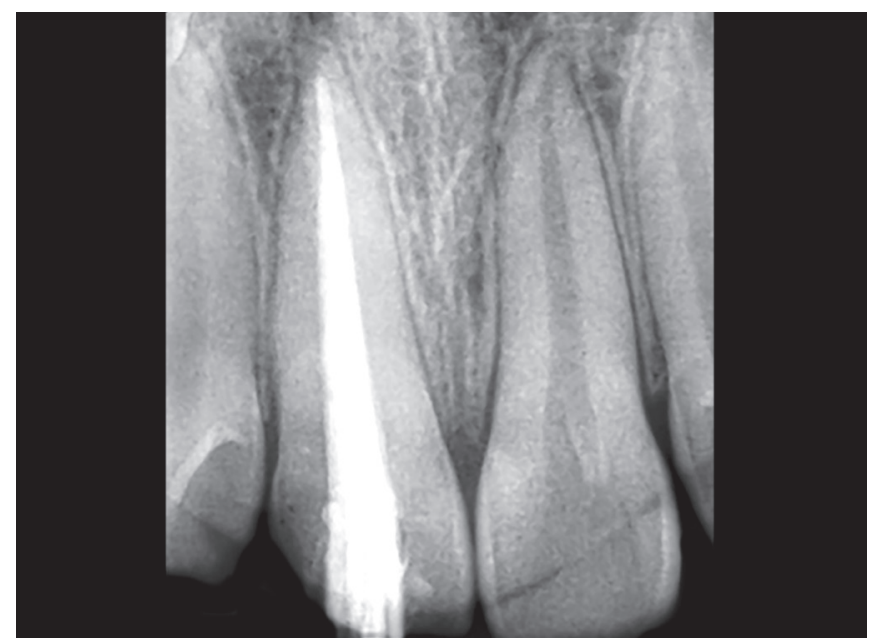

Fig. 3: Post obturation radiograph

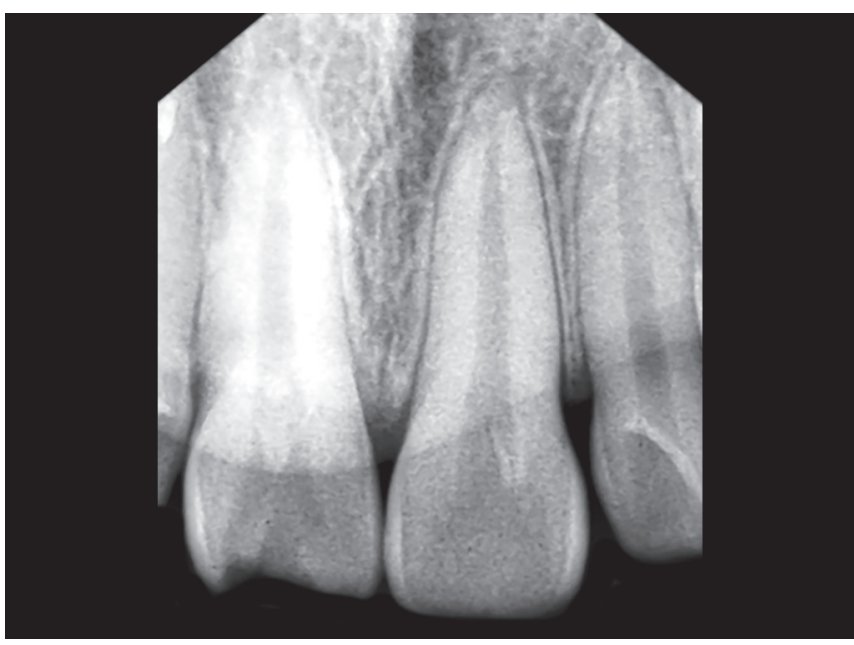

Fig. 2: Preoperative radiograph

was removed and the tooth was polymerized from both buccal and palatal aspects. During the final buildup, access cavity and missing portion of the tooth were restored with a composite resin (Te Econom plus Ivoclar Vivadent) to regain the tooth morphology (Fig. 7). The case was reviewed after 18 months (Figs 8 and 9).

\section{Discussion}

Anterior tooth trauma of a young patient is a tragic experience which requires immediate attention not only because of damage to dentition but also because of the psychological impact it may have on the patient and the parent. Various methods and technique were employed to restore fractured teeth which include pinretained resin, orthodontic band, stainless steel crown, porcelain jacket crown, and complex ceramic restorations. However, all these restorations require significant tooth preparation, they cannot be used in an emergency aesthetic situation.

Reattachment of the fractured tooth segment is one of the techniques for the immediate restoration of a fractured anterior tooth. It restores the morphological, functional, and aesthetic aspect of the dentition. The development of adhesive material creates a new perspective in the reconstruction of fractured teeth. It is now possible to achieve an excellent result with reattachment

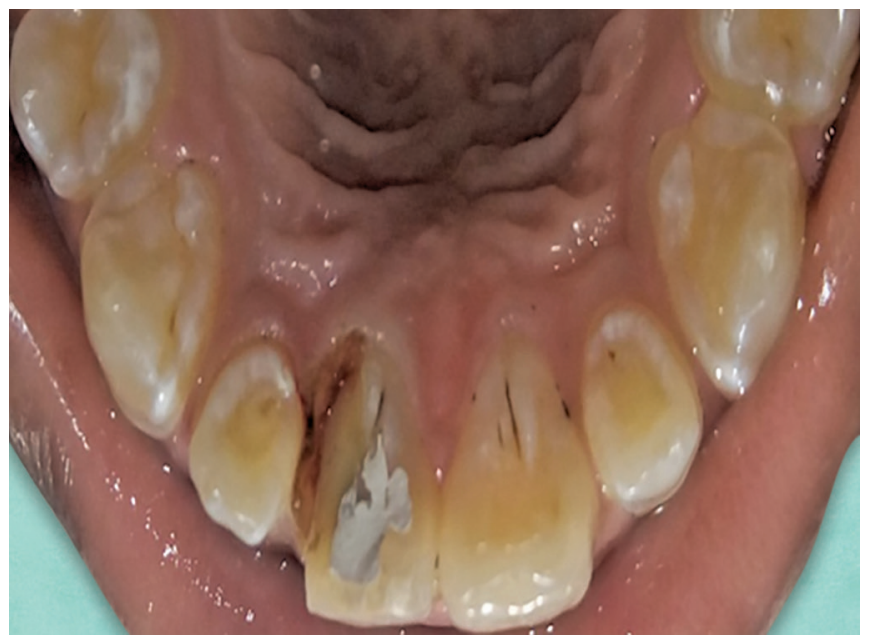

Fig. 4: After removal of fractured segment 


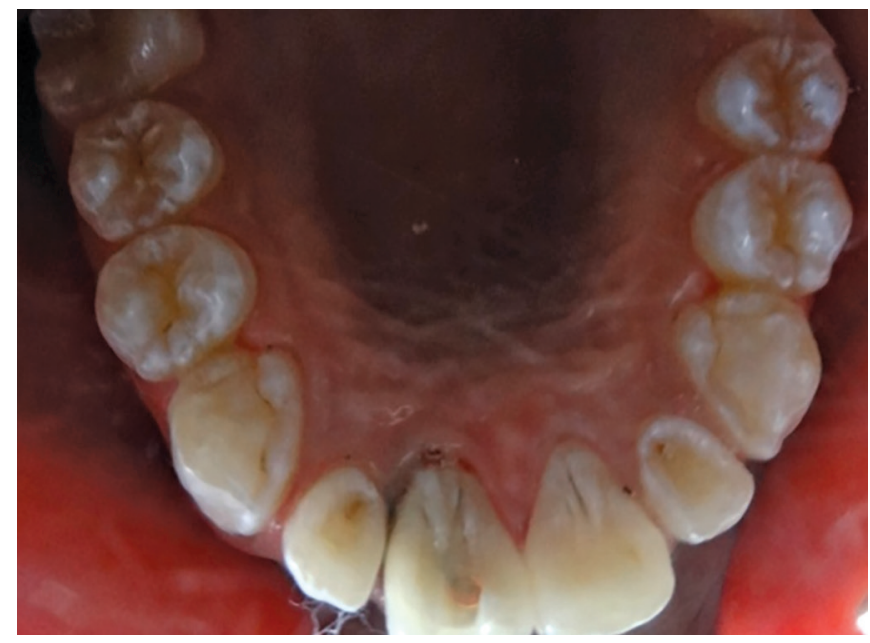

Fig. 5: After reattachment (palatal aspect)

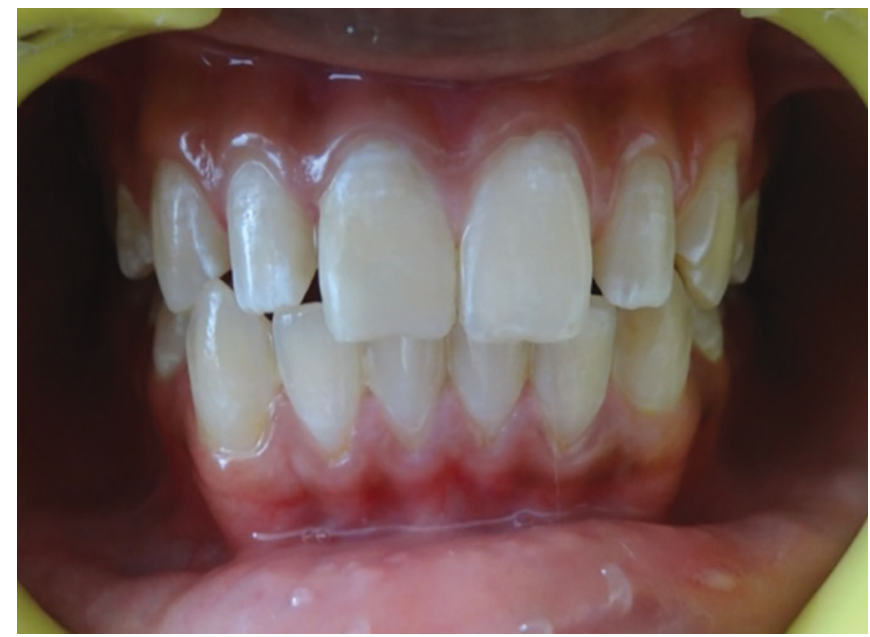

Fig. 7: Postoperative photograph

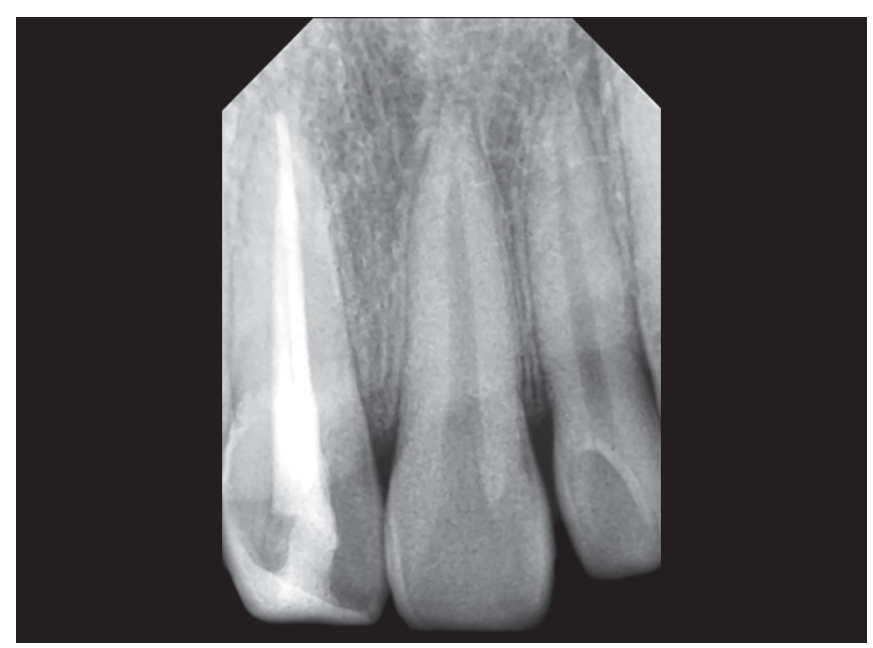

Fig. 9: Follow-up radiograph after 18 months

of tooth fragment, provided that the biological factors, materials, and technique are logically assessed and managed.

In the present case, the major portion of the coronal segment of the tooth was available and in good condition, fragment

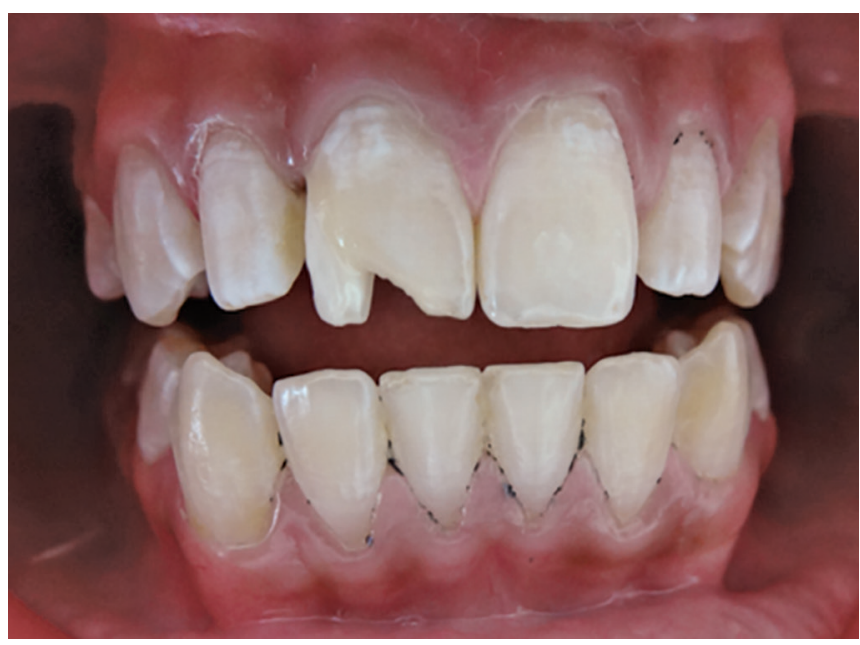

Fig. 6: After reattachment (labial aspect)

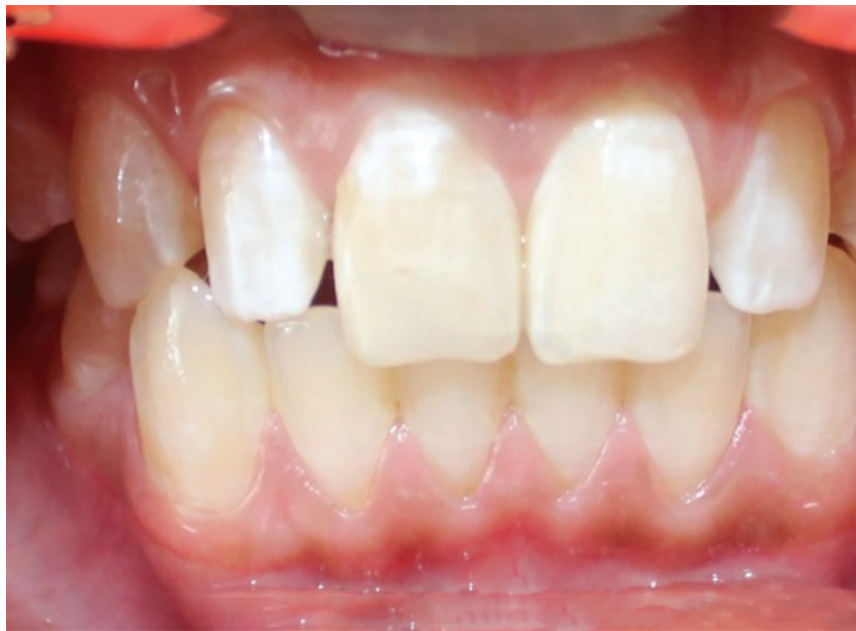

Fig. 8: Follow-up photograph after 18 months

reattachment was considered. In younger patient due to progressive eruption, and gingival margin instability, extensive restorations are not preferred. So considering the young age of the patient, reattachment was a better treatment option. Cavalleri and Zerman reported that the long-term prognosis for reattachment crown fragment appears to be better than for composite resin restorations. ${ }^{7}$

The success of reattachment depends on certain factors like the site of fracture, size of fractured remnant, periodontal status, pulpal involvement, maturity of the root formation, biological width invasion, occlusion, time and material used for reattachment, and use of post. The longer the fragment remains dehydrated, the tooth strength will be poor. Improvement of tooth resistance can be achieved by fragment rehydration. Dentin's dehydration causes collapse of collagen fibers and obstruction of adequate penetration of resin monomers leading to a poor adhesion between dentin and composite material. ${ }^{8}$ The fracture was reported to the department immediately after trauma and also the fractured segment was not separated. So the chances of dehydration of the fractured segment were very much less.

Since the fracture was complicated, endodontic treatment was done prior to the reattachment. In the present case, a periodontal crown lengthening procedure was done using laser gingivectomy in 
the palatal aspect of the 11 to expose the most apical extent of the fracture line which was below the alveolar bone. Reattachment was done on the same day since there was minimal bleeding following laser gingivectomy.

In the present case, reattachment of the tooth was done with no preparation technique. According to the studies of Shirani et al., ${ }^{9,10}$ fragment reattachment associated with the no preparation technique and adequate rehydration of the fragment presented higher bond strength values. Worthington et al. ${ }^{11}$ and Davari and Sadeghi ${ }^{12}$ reported that teeth with some types of additional preparations like bevels, overcontour, and internal grove do not present better bond strength than teeth reattached with the no preparation technique. $^{11}$

Resin cement was used as an intermediate restorative material for reattachment, considering that the dual-cure mechanism of the cement facilitates better bond strength. According to the study by Bhargava et al., ${ }^{13}$ where he compared adhesive system, luting cement resin, and nanoparticulate resin, as the intermediate restorative material, resin lutin cement showed the second best result.

Reattachment alone failed to give a favorable aesthetic result and an aesthetic composite buildup was done on the missing coronal portion. It provided an additional reinforcement to the reattached segment due to a strong composite bonding.

In cases of complicated fractures, when endodontic therapy is required, the space provided by the pulp chamber can be used as an inner reinforcement, thus, avoiding further preparation of the fractured tooth. ${ }^{14}$ So in the present case, space provided by the access cavity along with missing coronal portion restored with composite gave additional reinforcement to the reattachment. Similar types of reattachment cases were done by Kumar and Maheswari ${ }^{15}$ and Pathan and Gaddalay ${ }^{16}$ where they got better treatment outcome.

The patient was reviewed after 18 months and clinical and radiographic outcomes were satisfactory with normal contour and appearance.

\section{CONCLUSION}

Reattachment of the tooth is a viable treatment alternative to crowning in anterior tooth trauma of a young patient. If there is a missing coronal segment, reattachment has to be combined with composite contouring. The case report presents effective management of an oblique crown fracture managed with a singlevisit endodontic treatment followed by reattachment of tooth fragment. The missing coronal part was restored with composite which gives a better reinforcement to the fractured segment and the remaining tooth structure. It is a viable technique that restores function and esthetics with a very conservative approach and can be considered as a choice when treating a patient with coronal fractures of the anterior teeth especially in a young patient.

\section{References}

1. Hamilton FA, Hill FJ, et al. An investigation of dento-alveolar trauma and its treatment in an adolescent population. Part 1: the prevalence and incidence of injuries and the extent and adequacy of treatment received. Br Dent J 1997;182:91-95. DOI: 10.1038/sj.bdj.4809313.

2. Andreasen JO, Andreasen F, et al. Textbook and color atlas of traumatic injuries to the teeth, 3rd ed. St Louis (MO): Mosby; 1994.

3. Olsburgh S, Jacoby $\mathrm{T}$, et al. Crown fractures in the permanent dentition: pulpal and restorative considerations. Dent Traumatol 2002;18(3):103-115.

4. Reis A, Francci C, et al. Re-attachment of anterior fractured teeth: fracture strength using different techniques. Oper Dent 2001;26(3):287-294.

5. Baratieri LN, Ritter AV, et al. Tooth fragment reattachment: an alternative for restoration of fractured anterior teeth. Pract Periodontics Aesthet Dent 1998;10:115-125; quiz 127.

6. Andreasen FM, Noren JG, et al. Long term survival of fragment bonding in the treatment of fractured crowns. Quintessence Int 1995;26:669-681.

7. Cavalleri G, Zerman N. Traumatic crown fractures in permanent incisors with immature roots: a follow-up study. Endod Dent Traumatol 1995;11:294-296.

8. Perdigão J, Lopes M. Dentinbonding State of the art 1999. Compend Contin Educ Dent 1999;20:1151-1162.

9. Shirani F, Malekipour MR, et al. Hydration and dehydration periods of crown fragments prior to reattachment. Oper Dent 2012;37:501-508. DOI: 10.2341/10-130-L.

10. Shirani F, Malekipour MR, et al. Effect of storage environment on the bond strength of reattachment of crown fragments to fractured teeth. J Conserv Dent 2011;14:269-272. DOI: 10.4103/0972-0707.85813.

11. Worthington RB, Murchison DF, et al. Incisal edge reattachment: the effect of preparation utilization and design. Quintessence Int 1999;30:637-643.

12. Davari AR, Sadeghi M. Influence of different bonding agents and composite resins on fracture resistance of reattached incisal tooth fragment. J Dent 2014;15:6-14.

13. Bhargava M, Pandit IK, et al. An evaluation of various materials and tooth preparation designs used for reattachment of fractured incisors. Dent Traumatol 2010;26:409-412. DOI: 10.1111/j.16009657.2010.00913.x.

14. Diangelis AJ, Jungbluth $M$. Reattaching fractured tooth segments: an esthetic alternative. J Am Dent Assoc 1992;123(8):58-63. DOI: 10.14219/jada.archive.1992.0222.

15. Kumar $\mathrm{P}$, Maheswari $\mathrm{U}$. Reattachment of anterior teeth fragment, an aesthetic alternative: report of a case. J Oral Health Res 2010;1(3): 93-96.

16. Pathan ML, Gaddalay S. Reattachment of anterior teeth fragments: a case report. Int J Appl Dent Sci 2017;3(2):101-103. 